\title{
Epidemiología Del Trauma en Dos Hospitales de Primer Nivel de Atención Del Suroccidente de Colombia. Reporte Preliminar Del Registro Internacional de Trauma de la Sociedad Panamericana de Trauma
}

\author{
${ }^{1}$ Carlos Ordonez, ${ }^{2}$ Jaime Rubiano, ${ }^{3}$ Marisol Badiel, ${ }^{4}$ Luis F Pino, ${ }^{5}$ Fernando D Miñan-Arana, ${ }^{6}$ Jorge W Tejada \\ ${ }^{7}$ Mónica Morales, ${ }^{8}$ Juan C Puyana, ${ }^{9}$ Lina Mata, ${ }^{10}$ Michael Aboutanos, ${ }^{11}$ Cristina Vernaza, ${ }^{12}$ Rao R Ivatury
}

\section{RESUMEN}

Antecedentes: En Colombia las causas externas y violentas son la primera causa de muerte en menores de 44 años. El propósito de este estudio fue describir mecanismo, severidad y los desenlaces del trauma atendido en dos hospitales de tercer nivel del suroccidente colombiano.

Métodos: Entre Enero-2012 y Agosto-2012 se implementó el Registro Internacional de Trauma de la Sociedad Panamericana de Trauma (ITR-SPT) en dos hospitales civiles Nivel I en ${ }^{1,2,4,5,8,10}$ Surgeon, ${ }^{3}$ Vice Chairman, ${ }^{6,12}$ Doctor, ${ }^{7}$ Statistic Surgeon
${ }^{9}$ International Fellow, ${ }^{11}$ Professor (Emeritus)
${ }^{1}$ Chief of Trauma and Acute Care Surgery Department, Hospital
Universitario del Valle, Fundación Valle del Lili, Cali, Colombia
${ }^{2}$ Medical Director, Hospital Universitario del Valle, Cali
Colombia

${ }^{3}$ Department of Research, Clínica Universitaria Uribe, Uribe Cali, Colombia

${ }^{4}$ Director of the Emergency Department, Hospital Universitario del Valle, Cali, Colombia

${ }^{5}$ Trauma and Acute Care Fellow, Universidad del Valle, Cali Colombia

${ }^{6}$ Department of Basic Sciences, Universidad ICESI, Cali Colombia

${ }^{7}$ Department of Surgery, Hospital Universitario del Valle, Cali Colombia

${ }^{8}$ Department of Surgery, University of Pittsburgh, Pennsylvania USA

9ITSDP Coordinator, Division of Trauma Critical Care and Emergency Surgery, Virginia Commonwealth University Virginia, USA

${ }^{10}$ Chair, Division of Trauma, Critical Care and Emergency Surgery, Virginia Commonwealth University, Virginia, USA

${ }^{11}$ Division of Trauma, Critical Care and Emergency Surgery Commonwealth University Medical Center, Virginia, USA

${ }^{12}$ Colciencias Young Investigator, Department of Research Fundación Valle del Lili, Cali, Colombia

Corresponding Author: Carlos Ordonez, Surgeon, Department of Acute Care Surgery, Hospital Universitario del Valle Fundación Valle del Lili, Cali, Colombia, Phone: 572319090 e-mail: ordonezcarlosa@gmail.com
Cali, Colombia; uno de ellos de carácter público (PUB) y el otro privado (PRI). El ITR-SPT contiene información sociodemográfica, mecanismo del trauma, etiología, severidad según índice ISS (Injury Severity Score) y el estatus al egreso hospitalario.

Resultados: En el periodo de estudio se registraron 9.927 pacientes; edad promedio de $30 \pm 20,2$ años, el $67 \%$ fueron hombres. Las tres primeras causas de consulta fueron: caídas $(29,7 \%, n=2952)$, heridas penetrantes $(19,3 \%, n=1916)$ y accidentes de tránsito $(11,5 \%, n=1,142)$. Según la severidad del trauma, el 86,4\% $(n=8575)$ ingresaron con ISS <9; el $8 \%$ $(n=793$ ) con ISS 9 to 14 , y el $5,6 \%$ con ISS $\geq 15$ (PUB $8,6 \%$ vs PRI 3,6\%). La mortalidad general fue 3,4\% $(n=339)$ [PUB 6,6\% $(\mathrm{n}=268)$ vs PRI 1,2\% $(\mathrm{n}=71), \mathrm{p}<0,001]$.

Conclusión: En 7 meses, de medición ininterrumpida, en cerca de 10.000 pacientes, se identifica la tendencia real de la atención del trauma en hospitales de primer nivel. Las causas de consulta por trauma fueron similares en los dos hospitales. Parece ser más alta la mortalidad por el impacto de las heridas por armas de fuego en el hospital público luego del ajuste por severidad.

Palabras claves: Registro Internacional de Trauma, Sociedad Panamericana de Trauma (SPT), Caídas, Heridas penetrantes, Accidentes de tránsito.

How to cite this article: Ordonez C, Rubiano J, Badiel M, Pino LF, Miñan-Arana FD, Tejada JW, Morales M, Puyana JC, Mata L, Aboutanos M, Vernaza C, Ivatury RR. Epidemiología Del Trauma en Dos Hospitales de Primer Nivel de Atención Del Suroccidente de Colombia. Reporte Preliminar Del Registro Internacional de Trauma de la Sociedad Panamericana de Trauma. Panam J Trauma Crit Care Emerg Surg 2014;3(1): 11-15.

Source of support: Nil

Conflict of interest: None

\section{ABSTRACT}

Background: In Colombia, external and violent injuries are leading death causes on the population under 44 years. The purpose of this study was to describe and to compare trauma epidemiology, severity and mortality between a private and a public level I hospitals on Colombian Southwest.

Methods: Between January 2012 and August 2012, we implemented International Trauma Registry (ITR) from Panamerican Trauma Society (ITR-PTS) in two levels: one hospital in 
Cali, Colombia, one is a public hospital (PUB) and other is a private hospital (PRI). The registry includes sociodemographic information, trauma mechanism, severity by ISS (Injury Severity Score) and the clinical outcomes.

Results: In this period, 9,927 patients were registered, age mean was $30 \pm 20.2$ years, $67 \%$ were males. Most frequently causes of trauma were $29.7 \%$ falls, $19.3 \%$ penetrating injuries (PI) and $11.5 \%$ traffic accidents. By severity scores, $86.4 \%$ patients had ISS $<9.8 \%$ patients had ISS 9 to 14 and $5.6 \%$ had ISS $\geq 15$. The overall mortality rate $3.4 \%(n=339)$ [PUB $6.6 \%$ $(\mathrm{n}=268)$ vs PRI 1.2\% $(\mathrm{n}=71), \mathrm{p}<0.001]$.

Conclusion: Real time trauma registry implementation was feasible in the noninterrupted registry seven months period, almost 10,000 data patients were analyzed. These data are enough to establish preliminary tendencies, but still are insufficient to answer the factors related, by systematic data capture tools. We observed that trauma causes were similar in both hospitals. Mortality in severe patients with GSW was more frequently observed in public hospital. More studies and complementary analysis are required to assess patient's related factor in each hospital.

Keywords: Trauma registry, Panamerican Trauma Society, Falls, Penetrating injuries, Traffic accidents.

\section{INTRODUCCIÓN}

El trauma es una enfermedad que genera alrededor de 5 millones de muertes, ocupando la segunda causa de muerte a nivel mundial ${ }^{1}$ y generando altos gastos que varían según las regiones, por ejemplo en Estados Unidos el 10\% del gasto médico total se debió al trauma en el año $2000 .^{2}$ En América Latina la incidencia del trauma es del 11\% del total de la mortalidad asociada al trauma y Cali tuvo una tasa de mortalidad por lesiones personales de 82 por cien mil habitantes en el 2011. ${ }^{3}$

El trauma es una de las primeras causas de muerte en las personas de edades entre 14 to 45 años, es decir, la población joven y mas productiva desde el punto de vista laboral. ${ }^{1,3}$ En Colombia las causas externas y violentas son la primera causa de muerte en la población menor de 44 años. ${ }^{3,4}$

Debido a que el trauma es actualmente un problema de Salud Pública, considerado por la OMS como una epidemia, se han conformado los Centros de Trauma a nivel mundial con el objetivo primordial de disminuir la mortalidad ${ }^{2,5}$ así como los Sistema de Registro de Trauma (SRT) por medio de los cuáles se realiza la recolección sistemática de datos, obteniendo información objetiva acera de: la etiología del trauma, la severidad, el desenlace. ${ }^{2,3}$ A partir del análisis de ésta información se pueden tomar medidas a corto, mediano y largo plazo para mejorar la atención y sobrevida del paciente traumatizado. ${ }^{6-9}$

Por ello, el objetivo de este estudio es describir y comparar la etiología del trauma de dos hospitales de tercer nivel del suroccidente colombiano, de acuerdo a su mecanismo, severidad y los desenlaces.

\section{MATERIALES Y MÉTODOS}

A través de un convenio interinstitucional se logró la implementación del Registro Internacional de Trauma de la Sociedad Panamericana de Trauma (SPT) en dos hospitales civiles Nivel III en Cali, Colombia, uno de ellos de carácter público (PUB) y el otro privado (PRI). En el Registro se incluye información sociodemográfica, mecanismo del trauma, etiología, severidad con la estimación del índice (Injury Severity Score) (ISS) y el estatus de sobrevida al egreso hospitalario. Se presenta la información recolectada en tiempo real entre Enero 06 hasta Agosto 07 del 2012.

\section{Análisis Estadístico}

El registro exporta un archivo binario el cual se procesó en STATA ${ }^{\text {TM }} 12$ (StataCorp, Texas, USA). Se realizó un análisis exploratorio descriptivo; las variables categóricas son presentadas en proporciones y las comparaciones entre ellas se realizaron con prueba de chi cuadrado. Las variables continuas se expresan como media, mediana, promedios, las comparaciones entre ellas se realizaron con Prueba t de Student o prueba exacta de Fisher, según el caso. Como medida de asociación para mortalidad se estimó un riesgo relativo, estratificado por severidad. Se consideraron diferencias estadísticamente significativas si el valor de $\mathrm{p}<0,05$.

\section{RESULTADOS}

En el periodo de estudio se registraron 9,927 pacientes; el $67 \%$ fueron de sexo masculino, 70,6\% $(n=7,009)$ mayores de 18 años. Las tres primeras causas de consulta fueron: caídas $(29,7 \%, \mathrm{n}=2952)$, heridas penetrantes $(19,3 \%, \mathrm{n}=1916) \mathrm{y}$ accidentes de tránsito $(11,5 \%, \mathrm{n}=1,142)$, en la Figura $1 \mathrm{se}$ ilustran todas las causas de consulta para ambos hospitales. Según la severidad del trauma, el 86,4\% $(\mathrm{n}=8575)$ fueron pacientes con ISS $<9$; el $8 \%(n=793)$ con ISS 9-14, y el $5,6 \%$ tuvieron ISS $\geq 15$ (8,6\% PUB vs 3,6\% PRI), como se presenta en la Tabla 1. La mortalidad general fue 3.4\% $(\mathrm{n}=339)$. [PUB 6,6\% $(\mathrm{n}=268) v s$ PRI 1,2\%, $(\mathrm{n}=71), \mathrm{p}<$ $0,001]$. Según las tres primeras causas de consulta (Tabla 2) se analizaron un total de 7041 pacientes [PUB 50,9\%, (n= 3590) vs PRI 49,1\%, $(\mathrm{n}=3456)]$, la mortalidad general de este grupo fue mayor en el PUB [PUB 6,24\%, $(\mathrm{n}=224) v s$ PRI 1,97\%, $(n=68) ; p<0,001)$; en cuanto al índice severidad ISS, la mortalidad con ISS $>15$ fue significativamente mayor en el PUB [PUB 55,2\%, $(\mathrm{n}=185) v s$ PRI 33,3\%, $(\mathrm{n}=$ $64) ; p<0,002]$, y la mortalidad por heridas por arma de fuego en pacientes con ISS $>15$ fue mayor en el PUB [PUB 60,5\%, $(\mathrm{n}=133) v s$ PRI 37,7\%, $(\mathrm{n}=114) ; \mathrm{p}<0,0001]$. 


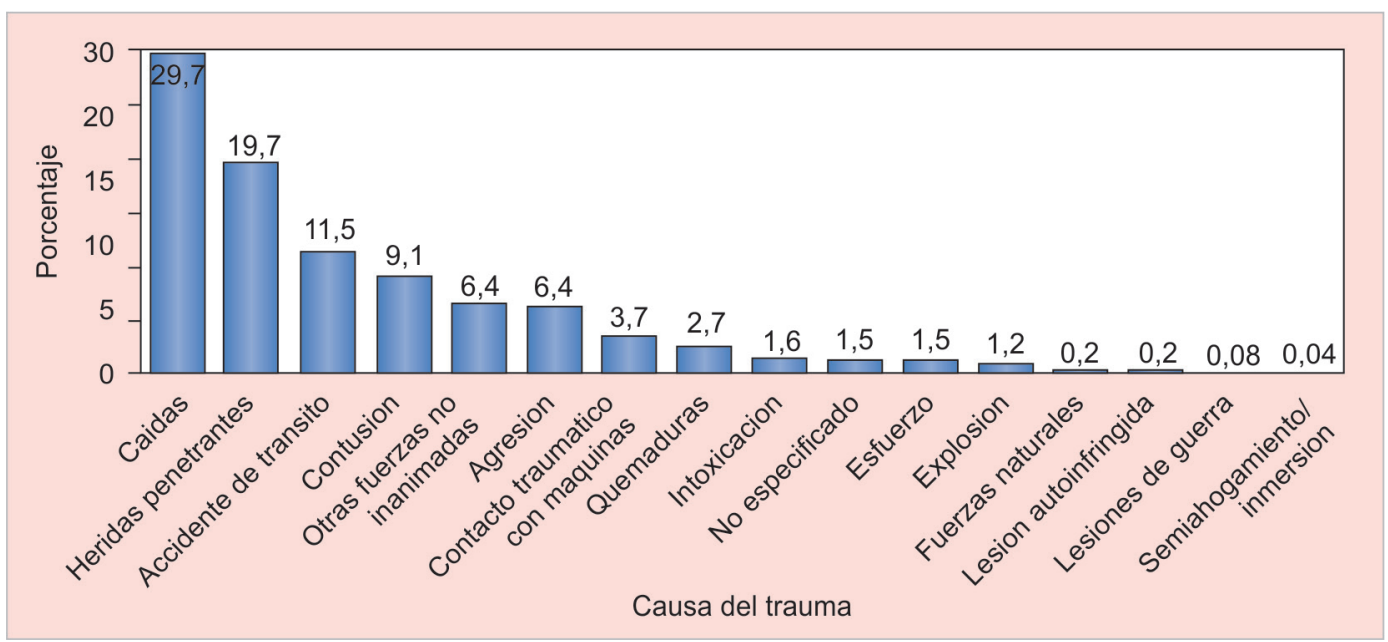

Figura 1: Distribución de todos los pacientes de la ciudad de Cali incluidos por los 2 hospitales en el Registro Internacional de Trauma

Tabla 1: Características socio-demográficas de la población registrada en cada hospital. Periodo enero-agosto 2012

\begin{tabular}{|c|c|c|c|}
\hline Características generales & Total $(n=9,927)$ & Privado $(n=5,909)$ & Publico $(n=4,018)$ \\
\hline Género, hombre, n (\%) & $6,655(67)$ & $3,702(62,7)$ & $2,953(73,5)$ \\
\hline Edad (años) ${ }^{* *}$ & $30 \pm 20,2$ & $29,2 \pm 18,8$ & $31,2 \pm 22,2$ \\
\hline \multicolumn{4}{|l|}{ Rangos de edad, n (\%) } \\
\hline$<18$ & $2,907(29,3)$ & $1,724(29,2)$ & $1,183(29,4)$ \\
\hline 18 a 44 & $4,851(48,9)$ & $3,020(51,1)$ & $1,831(45,6)$ \\
\hline 45 a 64 & $1,439(14,5)$ & $853(14,5)$ & $586(14,6)$ \\
\hline$\geq 65$ & $719(7,2)$ & $312(5,3)$ & $407(10,1)$ \\
\hline $\begin{array}{l}\text { Trauma relacionado con actividades laborales, } \\
\mathrm{n}(\%)\end{array}$ & $3034(30,6)$ & $2764(46,8)$ & $270(6,7)$ \\
\hline \multicolumn{4}{|l|}{ ISS, n (\%) } \\
\hline$<9$ & $8575(86,4)$ & $5488(92,9)$ & $3087(76,8)$ \\
\hline $9-14$ & $793(7,9)$ & $208(3,5)$ & $585(14,6)$ \\
\hline$\geq 15$ & $559(5,6)$ & $213(3,6)$ & $346(8,6)$ \\
\hline \multicolumn{4}{|l|}{ Hospitalización } \\
\hline >6 hrs observación, n (\%) & $4,274(43,1)$ & $608(10,3)$ & $3,666(91,2)$ \\
\hline Días, mediana $\left(\mathrm{RIC}^{\star}\right)$ & $1(0-3)$ & $2(1-6)$ & $1(0-2)$ \\
\hline Ingreso a UCI, n/N (\%) & $330 / 4,274(7,7)$ & $242 / 608(39,8)$ & $88 / 3,666(2,4)$ \\
\hline Estancia en UCI (días)* & $5(3-11)$ & $5(2-10)$ & $7(3-13)$ \\
\hline Mortalidad general, n (\%) & $339(3,4)$ & $71(1,2)$ & $268(6,1)$ \\
\hline Urgencias & $75(22,1)$ & $29(40,9)$ & $46(17,2)$ \\
\hline Hospitalización & $264(77,9)$ & $42(59,1)$ & $222(82,8)$ \\
\hline
\end{tabular}

*Mediana (RIC: rango intercuartílico); **Promedio \pm desviación estándar

Tabla 2: Clasificación según escala ISS para el hospital público por las tres causas más frecuentes de consulta a urgencias

\begin{tabular}{llll}
\hline \multicolumn{3}{l}{ Hospital público } & \\
\hline ISS & $<9$ & $9-14$ & $\geq 15$ \\
\hline Accidente de tránsito, $\mathrm{n}(\%)$ & $592(76,7)$ & $112(14,5)$ & $69(8,92)$ \\
Caídas, $\mathrm{n}(\%)$ & $1608(83,01)$ & $283(14,6)$ & $46(11,3)$ \\
Arma de fuego, $\mathrm{n}(\%)$ & $445(50,6)$ & $215(24,4)$ & $220(25)$ \\
Total & 2645 & 610 & 335 \\
Mortalidad para cada categoría de ISS, $\mathrm{n} / \mathrm{N}(\%)$ & $8 / 2645(0,3)$ & $31 / 610(5,1)$ & $185 / 335(55,2)$ \\
\hline
\end{tabular}




\section{DISCUSIÓN}

\section{El Trauma en Latinoamérica}

La experiencia en América Latina en la aplicación de un Registro de Trauma en tiempo real que se conoce es de Ecuador, donde se finalizó a los dos años por cese de recursos. ${ }^{10}$ No se encuentran más reportes con excepción de la aplicación del Registro Internacional de Trauma en la ciudad de Cali. ${ }^{3}$

\section{Aplicación del Registro de Trauma}

Fue posible implementar el Registro Internacional de Trauma en dos hospitales de Cali, pese a ser diferentes en su infraestructura en el manejo de la historia clínica. Se hace referencia a una Institución Pública (PUB) de referencia en el suroccidente del país y atención de pacientes pertenecientes principalmente al régimen subsidiado de salud; la Institución Privada (PRI) es sin ánimo de lucro, también centro de referencia y tiene convenio de atención al trauma de guerra, a este centro asisten más los pacientes del régimen contributivo. Ambas instituciones pertenecen al III nivel de atención en Colombia.

\section{Utilidad de los datos}

No hubo diferencias estadísticamente significativas entre los dos hospitales en cuanto a la causa de consulta. La mortalidad global fue 3,4\% $(n=339)$ La edad y género de esta población refleja la problemática de las lesiones de causa externa en Colombia, la mayor proporción de pacientes fueron hombres jóvenes entre 18 to 44 años $(48,9 \%, n=$ $4,851)$, datos que se correlacionan con lo reportados en algunos países de Latinoamérica, específicamente Chile y Argentina $^{2,7,11}$ (Tabla 1).

Según la etiología del trauma, en el presente estudio cerca del $60 \%$ de los traumas se distribuyen en tres causas principales: las caídas (29,7\%), en segundo lugar las heridas penetrantes $(19,3 \%)$ y tercero los accidentes de tránsito $(11,5 \%)$ (Tabla 2). Esto difiere con lo publicado por la OPS (Organización Panamericana de la Salud), donde indican que la primera causa de muerte en la población joven fue por accidentes de tránsito $(13,9 \%),{ }^{12,13} \mathrm{y}$ con datos obtenidos en algunos países de Latinoamérica, donde reportan que los accidentes de tránsito ocasionan la mayoría de muertes ${ }^{2,14}$
(Tablas 2 y 3). Así lo demuestra un reciente reporte en Argentina, por parte del programa CEDECEM trauma, donde se determinó que la mayor mortalidad en la población joven se debe a los accidente en moto. ${ }^{7}$

En los pacientes más graves, ISS $\geq 15$, las heridas penetrantes por arma de fuego representaron la mayor mortalidad $(51,9 \%, \mathrm{n}=176)$ este estudio se correlaciona con un estudio realizado en la ciudad de Medellín por Castrillón y cols, donde se determinó que la violencia (homicidio) fue la principal causa de muerte $(79,9 \%)$ y en segundo lugar los accidentes de tránsito $(12,2 \%)$ del total muertes registradas en el Instituto de Medicina Legal de esa ciudad, durante el 2006 hasta el 2009. ${ }^{1}$ Con datos de $2005,{ }^{14}$ la mayor tasa estimada de mortalidad por homicidio la tiene Colombia $(54,6 \times 100000$ hab. $)$, seguida de Venezuela $(32,4 \times 100000$ hab.) y Brasil $(31,0 \times 100000$ hab.) según un reporte de la OPS. Sin embargo la tasa estimada de mortalidad por causas externas en Colombia ha disminuido a partir del 2000 (105 × 100000 hab.) en relación al periodo de 1995 to 1999 que alcanzó a estar entre 120 y $140 \times 100000$ hab. según un reporte de la CEPAL (Comisión Económica para América Latina y El Caribe) para las Naciones Unidas en abril 2011 y la OPS. ${ }^{11,14}$

La mortalidad ajustada por severidad (ISS $>15$ ) y etiología es mayor en el hospital público, comparado con el privado, en el subgrupo de heridas por arma de fuego (PUB $\mathrm{n}=133$ vs PRI $\mathrm{n}=43, \mathrm{p}<0,0001)$ y con la mortalidad en general de los pacientes graves atendidos en dicho centro [PUB $(\mathrm{n}=185)$ vs PRI $(\mathrm{n}=64) ; \mathrm{p}<0,000002]$.

La diferencia de mortalidad obtenida entre las dos instituciones, en pacientes graves (ISS $>15$ ), puede ser explicada por las limitaciones en recursos físicos y económicos que tiene el PUB. En este hospital, hubo una hospitalización prolongada a mas de 6 horas [PUB 91,2\%, $(\mathrm{n}=3,666) v s$ PRI $10,3 \%(\mathrm{n}=608)]$, hay una falta de disponibilidad de camas en la UCI, reflejado en el porcentaje de ingreso a la UCI [PUB 2,4\%, $(\mathrm{n}=88) v s$ PRI 39,8\%, $(n=242)]$. Este mismo problema se presenta en otros países, algunos estudios muestran que el déficit de recursos de los hospitales públicos se asocia a una mayor mortalidad, no solo en pacientes con trauma sino también en patologías clínicas. ${ }^{15,16}$

Sin embargo los registros por institución muestran disparidades. Se puede observar que mientras el $64 \%$ de

Tabla 3: Clasificación según escala ISS para el hospital privado por las tres causas más frecuentes de consulta a urgencias

\begin{tabular}{llll}
\hline & & Hospital privado & \\
\hline ISS & $<9$ & $9-14$ & 215 \\
\hline Accidente de tránsito, $\mathrm{n}(\%)$ & $508(82,5)$ & $48(1,8)$ & $18,7)$ \\
Caídas, $\mathrm{n}(\%)$ & $2506(95,7)$ & $93(3,6)$ & $114(51,1)$ \\
Arma de fuego, n (\%) & $88(39,5)$ & $21(9,4)$ & 192 \\
Total & 3102 & 162 & $64 / 192(33,3)$ \\
Mortalidad para cada categoría & $2 / 3102(0,1)$ & $2 / 162(1,2)$ & \\
de ISS, n/N (\%) & & & \\
\hline
\end{tabular}


Epidemiología Del Trauma en Dos Hospitales de Primer Nivel de Atención Del Suroccidente de Colombia

Tabla 4: Distribución de los pacientes con ISS > 15 y heridas por arma de fuego según región anatómica afectada

\begin{tabular}{lllllll}
\hline Región anatómica afectada/mortalidad $(n / N)$ & $\begin{array}{l}\text { Todos los } \\
\text { pacientes }\end{array}$ & $\%$ & PUB & $\%$ & $P R I$ & $\%$ \\
\hline Cabeza & $87 / 117$ & 49,4 & $70 / 91$ & 52,6 & $17 / 26$ & 39,5 \\
Múltiple ( $\geq 2$ o más partes del cuerpo) & $71 / 152$ & 40,3 & $51 / 96$ & 38,3 & $20 / 56$ & 46,5 \\
Tórax & $10 / 25$ & 5,7 & $6 / 15$ & 4,5 & $4 / 10$ & 9,3 \\
Extremidades & $8 / 40$ & 2,8 & $6 / 18$ & 3 & $2 / 22$ & 2,32 \\
Abdomen & $5 / 24$ & 1,7 & $4 / 10$ & 1,5 & $1 / 14$ & 2,32 \\
\hline
\end{tabular}

PUB: Hospital público; PRI: Hospital privado

los casos de menor severidad son remitidos al PRI, el PUB recibe el $73,7 \%$ y $61,9 \%$ de los casos de mayores escalas de severidad respectivamente. Además se reciben 4 pacientes heridos por bala diarios en el Público vs 1 en el Privado.

Según los resultados, el 33,3\% de los pacientes más críticos terminan en fatalidad en el hospital privado mientras que en el hospital público es del 55,2\% y de igual manera lo es para menores niveles de gravedad. Sin embargo se deberían hacer futuras investigaciones para determinar de forma precisa las razones por la cuales se evidencia las diferencias entre las dos instituciones.

La región del cuerpo que tuvo mayor relación con mortalidad fue la cabeza $(49,4 \%)$ en pacientes con ISS $\geq$ 15 y heridos por arma de fuego, sin presentar diferencias estadísticas en las dos instituciones (PUB 52,6\% vs PRI $39,5 \%, p<0,65)$ (Tabla 4). Es posible que esta elevada mortalidad está asociada a las características anatómicofisiológicas propias del cerebro y cavidad craneana, así como las lesiones causadas por las armas de fuego.

\section{CONCLUSIÓN}

Fue factible la implementación del Registro de Trauma en tiempo real en dos tipos de hospitales nivel III. Disponer de una herramienta de captura electrónica en tiempo real, es el primer paso para establecer un sistema de información que permita identificar patrones y tendencias del trauma en la ciudad y así proponer estrategias para el manejo de estos pacientes al interior de cada institución para optimizar la utilización de los recursos y el sistema de referencia y también a nivel regional con la formulación de políticas públicas. Se encontró que las causas de consulta por trauma fueron similares en los dos hospitales, en edad y género y parece ser más alta la mortalidad en el hospital público luego del ajuste por severidad y etiología, en especial para las heridas por armas de fuego.

\section{REFERENCIAS}

1. Castrillón A, Escobar J, Gómez T, Morales J, et al. Causas de mortalidad en pacientes victimas de trauma entre los 15 a 45 años entre los años 2006 a 2009 en la ciudad de Medellín. http://www. bdigital.ces.edu.co:8080/dspace/bitstream/123456789/1009/2/c.

2. Medina E, Kaempffer A. Consideraciones epidemiológicas sobre los traumatismos en Chile. Rev Chilena de Cirugía 2007;59(3):175-218.
3. Ordoñez C, Pino L, Tejada J, Badiel M, Gutiérrez M, et al. Experiencia en dos Hospitales de Tercer nivel de atención del Suroccidente de Colombia en la aplicación del Registro Internacional de Trauma de la Sociedad Panamericana de Trauma (ITR/SPT-ITSDP). Rev Col Bras Cir 2012;39(4):255-262.

4. Cheng-Min H, Lunnen J, Miranda J, Hyder A. Traumatismo causados por el tránsito en países en desarrollo: Agenda de investigación y de acción. Rev Peru Med Exp Salus Publica 2010;27(2):243-247.

5. Odriozola F, Azaldegui F. Impacto de la implantación de un sistema de cuidado traumatológico en la mortalidad del trauma severo. Med Intensiva 2006;30(7).

6. Costanti C, Dominguez C, Cardoso R, Nogueira L. Preventable trauma death. Rev Saúde Pública 2012;46(2):1-8.

7. Programa CEDECEM. Informe sobre lesiones traumáticas vinculadas al transporte http://www.observatoriovial. seguridadvial.gov.ar/documentos/ops/2012-08-09-informecedecem-trauma.pdf.

8. CEPAL, América latina y el Caribe. Observatorio demográfico No.4. http://www.eclac.org/publicaciones/xml/5/33265/OD-4mortalidadenAL.

9. López E. Debate en Medicina en Medicina Intensiva Asistencia al Paciente Traumatizado: Centros de Trauma vs Hospital General, Pro Centro de Trauma. Med Intensiva 2010;34(3):188-193.

10. Mora F, Aboutanos M, Mora, et al. Uso de la Tecnología en la creación de un sistema de Trauma en Ecuador. Revista de Salud 2006;2(8).

11. Mariachiara Di Cesare. El perfil epidemiológico de América Latina y el Caribe: desafíos, límites y acciones. Naciones Unidas, abril de 2011. http://www.eclac.org/publicaciones/xml/9/44309/ lcw395.pdf.

12. Lugo L, García H, Cano B, Lasprilla AJ, Alcaraz O. 2013 Multicentric study of epidemiological and clinical characteristics of persons injured in motor vehicle accidents in Medellín, Colombia 2009-2010;44(2):100-107. Available at: http:// colombiamedica.univalle.edu.co/index.php/comedica/article/ view/1106

13. Patton G, Coffey C, Sawyer S, et al. Global patterns of mortality in young people: a systematic analysis of population health data. Lancet 2009;374:881-892.

14. Organización Panamericana de la Salud. Situación de salud en la américas, indicadores básicos 2005. http://www.paho.org/ spanish/dd/ais/coredata.htm

15. Morris M, Lacopetta B, Platell C. Comparing survival outcomes for patients with colorectal cancer treated in public and private hospitals. https://www.mja.com.au/journal/2007/186/6/ comparing-survival-outcomes-patients-colorectal-cancertreated-public-and-private.

16. Permanyer G, Brotons C, Ribera A. Desigual perfil clínico, calidad de vida y mortalidad hospitalaria en pacientes operados de injerto aortocoronario en centros públicos y privados de Cataluña. Rev Esp Cardiol 1998;51. 